\title{
Efficient Resource Allocation for IEEE 802.15.3(a) Ad Hoc Networks
}

\author{
Yi-Hsien Tseng ${ }^{1}$, Hsiao-Kuang $\mathrm{Wu}^{2}$, Kuen-Long Shieh ${ }^{1}$, and Gen-Huey Chen ${ }^{1}$ \\ 1 Department of Computer Science and Information Engineering, \\ National Taiwan University, Taipei, Taiwan \\ 2 Department of Computer Science and Information Engineering, \\ National Central University, Chung-Li, Taiwan
}

\begin{abstract}
IEEE 802.15.3(a) is working to design a higher speed PHY enhancement amendment to 802.15.3 such as ultra-wideband which can provide precise timing and location information. This paper proposes an effective resource allocation mechanism which can guarantee the QoS properties of multimedia traffics and provide higher channel utilization by the aid of location information. The proposed mechanism, which is executed by the piconet coordinator, consists of three components: grouping, call admission control, and best effort traffic maximization.

The grouping component groups non-interfering traffics together so that they are allowed to be transmitted simultaneously. The problem of grouping is shown equivalent to the graph coloring problem for which a lot of algorithms are available. The CAC component is responsible for QoS guarantee. The best effort traffic maximization component attempts to maximize the total throughput of best effort traffics. The maximization problem is formulated as a linear programming. Finally, simulation results verify the ability of QoS guarantee and higher channel utilization.
\end{abstract}

\section{Introduction}

IEEE 802.15.3 [1], which specified both the physical layer and MAC layer, was proposed for short range communication in wireless personal area networks (WPANs) with the advantages of low power consumption, low cost and low complexity. The physical layer can support data rate up to $55 \mathrm{Mbps}$. Then, for providing higher data rate, the IEEE 802.15 Task Group 3a (IEEE 802.15.3a) adopted ultra-wideband (UWB) technology and proposed new physical layer specifications whose data rate ranged from $110 \mathrm{Mbps}$ to $480 \mathrm{Mbps}$. IEEE 802.15.3a could be used by the MAC layer of IEEE 802.15.3.

UWB technology [14 had been studied from the 1960's to the 1990's by the United States military for its use in modern RADAR. Later, the Federal Communications Commission (FCC) announced a Notice of Proposed Rule Making (NPRM) 2 to address rules for UWB emission. Furthermore, the FCC commissioners unanimously approved limited UWB uses in February 2002. UWB devices either had their fractional bandwidths greater than 0.25 or occupied 1.5 $\mathrm{GHz}$ or more of the spectrum. Instead of using the traditional RF carriers, UWB 
transmitters emitted a series of short pulses for data transmission. Each short pulse, also called a monocycle, is equivalent to a single sine wave. The duration of a UWB monocycle varies from 0.2 to 1.5 nanoseconds.

The IEEE 802.15.3 MAC protocol [1, which was designed for high data rate WPAN, can provide a reliable QoS supporting framework. The elementary topological unit for the IEEE 802.15.3 MAC layer is a piconet, which is a wireless ad hoc data communications system in essence. There are a number of independent data devices (DEVs) contained in a piconet that are allowed to exchange frames directly with each other. The master/slave relationship was adopted for these DEVs; a particular DEV, named piconet coordinator (PNC), acts as the master and the others are slaves.

Although the IEEE 802.15.3 MAC layer can provide a QoS supporting framework, it does not specify the functions of scheduling and admission control. So, an efficient scheduling method and a smart admission control strategy are still needed, in order to ensure smooth multi-service deliveries such as distinct bandwidth and delay requirements. They are intended to determine when a DEV can start transmission and decide which channel time requests are admitted.

With the location information provided by UWB technology and the transmission power control (TPC) supported by IEEE 802.15.3, a resource allocation mechanism for high-speed WPANs is proposed in this paper. This resource allocation mechanism contains an effective scheduling method and an effective admission control strategy. With this mechanism, the system throughput can be maximized and the channel utilization can be enhanced, while the QoS requirements are satisfied.

The rest of this paper is organized as follows. Related work is introduced in the next section. In Section 3, the IEEE 802.15.3 MAC protocol is briefly reviewed. The resource allocation mechanism is described in Section 4. Simulation and comparison results are shown in Section 5. Finally, this paper concludes with some remarks in Section 6.

\section{Related Work}

In this section, scheduling algorithms for both wireline and wireless networks are briefly reviewed. Basically, they are modifications or extensions of the well-known generalized processor sharing (GPS) 26]. GPS, which was originally proposed for wireline networks, is also known as weighted fair queueing (WFQ) model [11. We note that GPS is an ideal fair scheduling model. In order for GPS to be implemented in a TDMA packet network, in which packets are not infinitely divisible and one session is served at a time, a practical packet-by-packet GPS (PGPS) was proposed in [26].

PGPS assigned each incoming packet a timestamp $F_{i}^{k}$ which was computed by $F_{i}^{k}=S_{i}^{k}+L_{i}^{k} / R_{i}$ and $S_{i}^{k}=\max \left\{v\left(a_{i}^{k}\right), F_{i}^{k-1}\right\}$, where $R_{i}$ is the transmission rate of session $i$ and $F_{i}^{k}, S_{i}^{k}, L_{i}^{k}, a_{i}^{k}$ are the virtual finish time, virtual start time, packet length and arrival time of the $k$-th packet of session $i$, respectively. 
Besides, $v(t)$ is a virtual time function which was calculated according to $d v(t) / d t=$

$C /\left(\sum_{i \in B(t)} R_{i}\right)$, where $C$ is the fixed channel rate and $B(t)$ is the set of backlogged sessions at time $t$. Incoming packets were served in increasing order of their timestamps. There were other modified versions of GPS, i.e., worst-case fair weighted fair queueing $\left(\mathrm{WF}^{2} \mathrm{Q}\right)$ [8, start-time fair queueing (STFQ) [15] and self-clocked fair queueing (SCFQ) [16]. More scheduling algorithms for wireline networks can be found in [29].

Since all GPS-based scheduling algorithms that were mentioned above assumed error-free channels, it is improper to implement them in wireless networks. Compared with wireline networks, wireless networks incline to a burst of location-dependent channel errors due to interference, fade and multipath effect. An additional compensation model should be added to these GPS-based scheduling algorithms, in order to deal with channel errors in wireless networks. The purpose of the compensation model is to enable lagging flows to reclaim services that were lost due to channel errors.

There were some scheduling algorithms proposed for wireless networks, e.g., channel state dependent packet scheduling algorithm (CSDPS) 7], idealized wireless fair queueing algorithm (IWFQ) 23], channel independent fair queueing algorithm (CIF-Q) 25], server based fairness algorithm (SBFA) 28, and wireless fair service algorithm (WFS) 23. They all combined GPS-based scheduling algorithms with compensation models. GPS-based scheduling algorithms can guarantee QoS properties.

At the first glance, it seems possible to directly adapt GPS-based scheduling algorithms to the IEEE 802.15.3 MAC protocol. It was stated in 9 that when the network was at a high data rate, GPS-based scheduling algorithms might cause a bottleneck which was incurred by the heavy computation required for evaluating virtual time tags of packets and then sorting them. So, it is rather difficult to implement GPS- based scheduling algorithms in the IEEE 802.15.3 network, because DEVs have low computing power.

In this paper, a new scheduling algorithm for the IEEE 802.15.3 MAC protocol is proposed in which DEVs need not calculate virtual time tags. DEVs only have to inform the PNC of bandwidth requirements and delay limitations of pending traffics via request messages. With these information, the scheduling algorithm can guarantee QoS properties. These request messages do not involve heavy computation.

\section{Review of IEEE 802.15.3 MAC Protocol}

A piconet contains $236 \mathrm{DEVs}$ at most. At the initialization of a piconet, one of the DEVs is elected to be the PNC. The PNC broadcasts the current status of DEVs periodically via beacons. DEVs are also aware of newly entering DEVs by beacons. When the PNC finds a more capable DEV, it hands over the control of the piconet to the DEV. That is, the old PNC is no longer the PNC and the DEV acts as the new PNC. Timing for a piconet is realized by superframes whose three parts are briefly described below (also refer to Figure 1). 


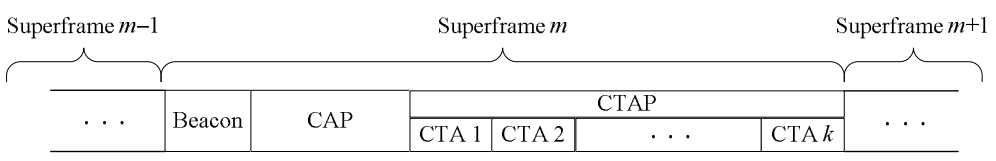

Fig. 1. A superframe and its three parts

- Beacon. It contains a beacon frame and announcement commands (see [1]) that are sent by the PNC as a beacon extension. Its purpose is to set timing allocations and to distribute management information over the piconet.

- CAP. It is optional. If existing, it allows the PNC and DEVs to change commands and/or asynchronous data in a contention manner (CSMA/CA).

- CTAP. It consists of some channel time allocations (CTAs), which are reserved for the PNC and DEVs to change commands, isochronous streams and asynchronous data in a non-contention manner (TDMA).

The durations of the CAP and the CTAP, which are determined by the PNC according to the channel time needs of current requests, may vary in different superframes. DEVs are allowed to change data during the CAP or the CTAP. The CAP is suitable for small and non time- critical data transmissions in a contention manner. On the other hand, the CTAP is suitable for data transmissions with QoS guaranteed. When a DEV intends to transmit data during the CTAP, it has to send a request message to the PNC first. The PNC then decides whether the request can be accepted or not according to the available time in the superframe. If accepted, the PNC will allocate enough CTAs for the DEV and announce this allocation in the next beacon.

The 802.15.3 MAC protocol provides a functionality of controlling transmitter power. The ability to control power enables DEVs to minimize the interference with other wireless networks that share the same channel and to decrease the power consumption. There are two methods for controlling transmitter power. One allows the PNC to set a maximum level of transmission power for the CAP and the beacon. The other, which is adopted by this paper, allows DEVs to enhance or reduce the transmission power.

\section{A Resource Allocation Mechanism}

In this section, an efficient resource allocation mechanism for IEEE 802.15.3(a) networks is proposed. We do not deal with any particular time-critical traffics (such as CBR and real-time VBR) and non-time-critical traffics (such as nonreal-time VBR, UBR and ABR). Instead, we classify traffics into two categories: real-time traffics (RTs) and non- real-time traffics (NRTs). Using the method of [10] together with UWB technology, the PNC can compute relative positions of DEVs. They are also aware of the relative position of each other via beacons.

The IEEE 802.15.3 system architecture equipped with the proposed mechanism is shown in Figure 2. The proposed mechanism, which is implemented 


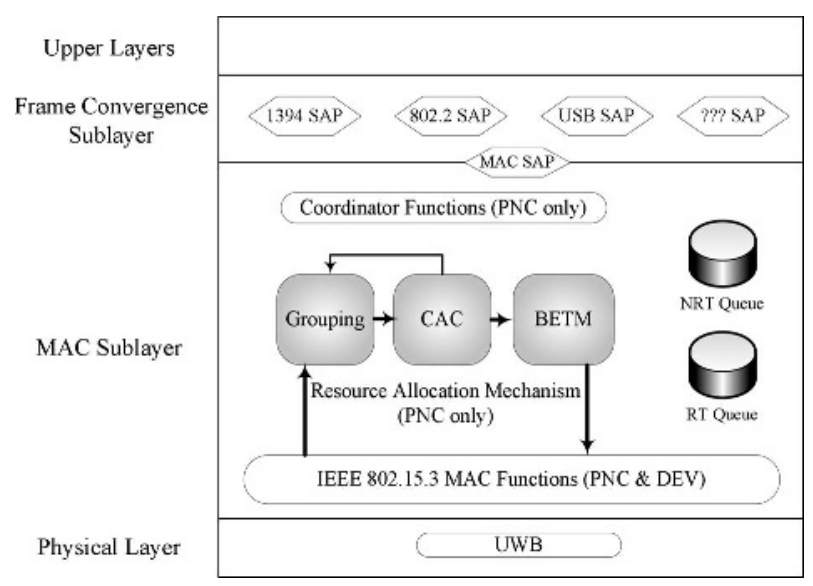

Fig. 2. The IEEE 802.15.3 system architecture with the proposed resource allocation mechanism

in the PNC only, contains three components: grouping, call admission control (CAC) and best effort traffic maximization (BETM). For each superframe, the grouping component determines the number of CTAs contained in it. The CAC component determines which traffic requests can be accepted. The BETM component determines the length of each CTA, the length of the superframe and the duration of each traffic request. The PNC informs all DEVs of these via beacons.

When a DEV wants to transmit data, it sends a request message to the PNC during the CAP. At the end of the CAP, the PNC may receive a number of request messages and the grouping component then forms these requests into groups so that non-interfering requests belong to the same group (one request may belong to more than one group). The number of distinct groups determines the number of CTAs required and traffics belonging to the same group can be transmitted simultaneously by dynamically adjusting transmission power. The problem of grouping is equivalent to the graph coloring problem [27].

The CAC component is responsible for checking whether there is enough time for transmitting RTs and whether their QoS requirements can be satisfied. If not, the $\mathrm{CAC}$ component will reject some $\mathrm{RT}$ requests and the grouping component will be invoked again with the rejected RT requests excluded. Otherwise, the BETM component then takes over whose objective is to maximize the total throughput of all NRTs subject to some constraints. At the same time, the length of each CTA can be determined and the channel utilization can be enhanced. The maximization problem can be formulated as a linear programming [20]. In the rest of this section, the three components are described in detail.

\subsection{Grouping}

The chief purpose of grouping is spatial reuse. Figure 3 further illustrates the concept. Since the transmission between a TV and a video device does not inter- 


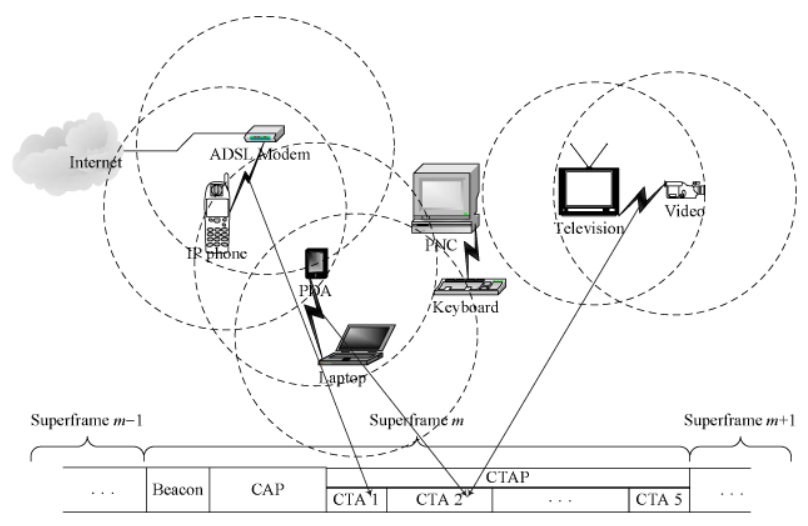

Fig. 3. The concept of grouping

ferewith the transmission between a PDA and a laptop, they can be scheduled in CTA 2. In this way, channel utilization is enhanced.

In traditional TDMA systems, one transmission was permitted at a time. That is, only one traffic request could be accepted for each CTA. By exploiting location information and transmission power control, which are supported by IEEE 802.15.3, more than one traffic request can be scheduled in a CTA. Now that every two neighboring DEVs can compute their distance, they can adopt minimal level of transmission power so that they can hear each other. Consequently, less interference occurs within a piconet, and more non-interfering traffics can be transmitted simultaneously.

In order for non-interfering traffics to be transmitted during the same time period, the PNC must be aware of all interference. DEVs having pending traffics are required to send request messages to the PNC during the CAP. Since these request messages contain the identifiers of source and destination DEVs, the PNC can easily determine if there is interference among these traffics by computing the distance of any two DEVs.

The grouping component attempts to schedule non-interfering traffic requests in the same CTA, with the objective of minimizing the number of CTAs in a superframe. With the same traffic requests, fewer CTAs are likely to have a better channel utilization. The problem of grouping can be formally described as follows.

The Problem of Grouping. Given a set $R=\left\{r_{1}, r_{2}, \ldots, r_{n}\right\}$ of traffic requests and a set $T=\left\{t_{1}, t_{2}, \ldots, t_{k}\right\}$ of CTAs, determine a mapping $F$ from $R$ to $T$ so that $|F(R)|$ is minimized, subject to the constraint that $F\left(r_{i}\right)=F\left(r_{j}\right)$ implies no interference between $r_{i}$ and $r_{j}$ for all $1 \leq i \leq n$ and $1 \leq j \leq n$.

The following theorem shows that the problem of grouping is equivalent to the graph coloring problem as described below.

The Graph Coloring Problem. Given the vertex set $V=\left\{v_{1}, v_{2}, \ldots, v_{n}\right\}$ of a graph $G$ and a set $C=\left\{c_{1}, c_{2}, \ldots, c_{k}\right\}$ of $k$ distinct colors, determine a mapping $H$ from 
$V$ to $C$ so that $|H(V)|$ is minimized, subject to the constraint that $H\left(v_{i}\right)=H\left(v_{j}\right)$ implies that $v_{i}$ and $v_{j}$ are not adjacent in $G$ for all $1 \leq i \leq n$ and $1 \leq j \leq n$. A feasible mapping $H$ satisfying the constraint is called a $|H(V)|$-coloring of $G$.

Theorem 1. The problem of grouping is equivalent to the graph coloring problem.

Proof. We first show that each instance of the problem of grouping can be transformed into an instance of the graph coloring problem. Consider $R=$ $\left\{r_{1}, r_{2}, \ldots, r_{n}\right\}$ and $T=\left\{t_{1}, t_{2}, \ldots, t_{k}\right\}$ an arbitrary instance, denoted by $I$, of the problem of grouping. Denote each $r_{i}$ by a vertex $v_{i}$ and each interference between $r_{i}$ and $r_{j}$ by an edge $\left(v_{i}, v_{j}\right)$. The resulting graph is denoted by $G=(V, E)$, where $V=\left\{v_{1}, v_{2}, \ldots, v_{n}\right\}$.

Suppose that $F: R \rightarrow T$ is a feasible mapping for $I$, i.e., $F\left(r_{i}\right)=F\left(r_{j}\right)$ implies no interference between $r_{i}$ and $r_{j} . F$ has the following property for $G: F\left(v_{i}\right)=F\left(v_{j}\right)$ implies that $v_{i}$ and $v_{j}$ are not adjacent in $G$. Moreover, there exists a feasible $F$ for $I$ if and only if $G$ has a $|F(R)|$-coloring, imaging that there is a one-to-one correspondence from $T=\left\{t_{1}, t_{2}, \ldots, t_{k}\right\}$ to $C=\left\{c_{1}, c_{2}, \ldots, c_{k}\right\}$. Hence, $G$ is an instance of the graph coloring problem.

Similarly, each instance of the graph coloring problem can be transformed into an instance of the problem of grouping so that the former has a $|H(V)|$ coloring if and only if there exists a feasible mapping $F$ with $|F(R)|=|H(V)|$ for the latter.

With Theorem 1, both the problem of grouping and the graph coloring problem are referred to interchangeably from now on. The graph coloring problem is known to be NP-hard [22. A graph $G$ is $k$-colorable if there is a $k$-coloring of $G$. The minimum $k$ so that $G$ is $k$-colorable is called the chromaticnumber of $G$. There are some exact algorithms and approximation algorithms for the graph coloring problem. Most of exact algorithms are based on implicit enumeration. The well-known DSATUR algorithm [6] is one of them. Differently, Méndez Diaz and Zabala proposed an LP-based Branch-and- Cut algorithm in 24] for the graph coloring problem.

On the other hand, an approximation algorithm for coloring an $n$-vertex graph $G$ were proposed in [5] [17] [19. Interested readers may consult 24] [5] [19] for more solution methods, exact and approximate, to the graph coloring problem.

\section{2 $\quad \mathrm{CAC}$}

Since the length of a superframe is limited $(\leq 65535 \mu \mathrm{s})$, some requests should be rejected if the available channel time is insufficient. For example, when the total time required by the requests exceeds the maximally allowable length of CTAP, some requests should be rejected. The CAC component is responsible for resource management so that QoS properties can be guaranteed.

The CAC component deals with only RT requests because they are associated with delay limitations and bandwidth requirements. The PNC computes an 
upper bound on the length of a superframe and lower bounds on the lengths of CTAs for the purpose of admission control. If the summation of all lower bounds for CTAs is greater than the upper bound, the PNC will reject an RT request and then invoke the grouping component again. After regrouping, fewer CTAs may be used.

The upper bound is determined by two factors: the maximal length $(65535 \mu \mathrm{s})$ specified by the IEEE 802.15.3 standard and delay limitations associated with $\mathrm{RT}$ requests. The upper bound is computed as the minimum of the maximal length and those delay limitations. For example, the upper bound is $48 \mathrm{~ms}$ if there are three RT requests whose delay limitations are $78 \mathrm{~ms}, 65 \mathrm{~ms}$ and $48 \mathrm{~ms}$, respectively. By keeping the length of the superframe below the upper bound, all delay requirements can be satisfied.

On the other hand, the lower bound for a CTA is computed as the maximal bandwidth requirement of the RT requests scheduled in it. If the CTA contains only NRT requests, the lower bound is set to zero. For example, refer to Figure 4 where there are three RT requests scheduled in CTA 1 and CTA 2. Since their bandwidths are $44 \mathrm{~ms}, 32 \mathrm{~ms}$ and $22 \mathrm{~ms}$, the lower bounds for CTA 1 and CTA 2 are $44 \mathrm{~ms}$ and $22 \mathrm{~ms}$, respectively. The lower bound for CTA $k$ is zero because it contains no RT request.

Figure 4 also illustrates the concept of CAC. The upper bound represents the maximally allowable length of the superframe. If the summation of all lower bounds is greater than the upper bound, then the available channel time is insufficient for these RT requests and so an RT request should be rejected. The rejected $\mathrm{RT}$ request is the one interfering with the most $\mathrm{RT}$ requests. If there is more than one candidate, select the one with the smallest delay limitation. The grouping component is then invoked again with the rejected RT request excluded.

If the summation of all lower bounds is not greater than the upper bound, the BETM component then takes over, which is detailed below.

\subsection{BETM}

After the CAC component, the QoS requirements of all RTs are guaranteed. In this section, we intend to maximize the total throughput of all NRTs based on a reassignment of requests to CTAs. The maximization is subject to the

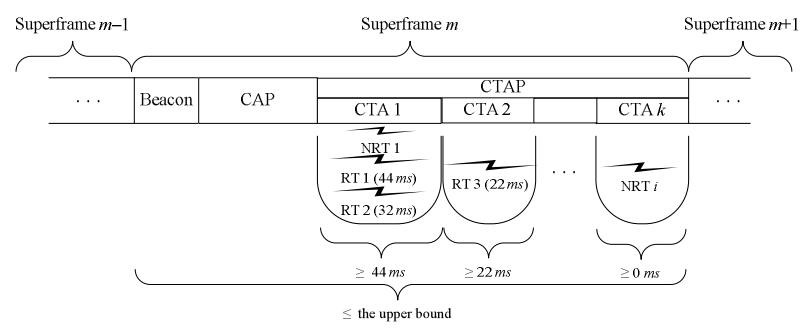

Fig. 4. The concept of CAC 
constraints induced by the upper bound and lower bounds of Section 4.2. Recall that after the grouping component, each request, RT or NRT, is assigned to a unique CTA. Now a reassignment is to be made in order to increase the total throughput. Each NRT request in the reassignment may be assigned to extra CTAs, whereas each RT request remains the same assignment as before.

The reassignment is described as follows. Suppose that there are $m$ NRT requests, denoted by $r_{\phi(1)}, r_{\phi(2)}, \ldots, r_{\phi(m)}$, and each $r_{\phi(i)}(1 \leq i \leq m)$ interferes with $p_{\phi(i)}$ NRT requests. First, arrange these $m$ NRT requests in a nondecreasing sequence of $p_{\phi(i)}$ 's. Then, for each NRT request, say $r_{\phi(i)}$, in the sequence, sequentially perform the following: schedule $r_{\phi(i)}$ in a new CTA if it does not interfere with any (RT or NRT) request contained in the CTA.

Figure 5 shows an example. Suppose that there are two RT requests, denoted by RT 1 and RT 2, and three NRT requests, denoted by NRT 1, NRT 2 and NRT 3. The interference is expressed with a graph, as shown in Figure 5(a), whose vertices represent the requests and whose edges represent the interference among them. The assignment of the requests to CTAs after the grouping component is shown in Figure 5(b). The reassignment of NRT requests can be performed in the sequence of NRT 3, NRT 2 and NRT 1, and the result is shown in Figure 5(c). The reassignment schedules NRT 2 in CTA 3 and NRT 3 in CTA 1 and CTA 3, additionally. After the reassignment, the total throughput of all NRTs can be enhanced.

Let $T_{i}$ be the set of CTAs where $r_{\phi(i)}$ is scheduled after the reassignment. For convenience, we use $|\mathrm{CTA} j|$ to denote the time length of CTA $j$ and $\left|r_{\phi(i)}\right|$ to denote the time length required by $r_{\phi(i)}$. Now that $r_{\phi(i)}$ is scheduled in the CTAs of $T_{i},\left|r_{\phi(i)}\right|$ is distributed over them. We use $\left|r_{\phi(i)}\right|^{(q)}$ to denote the portion of $\left|r_{\phi(i)}\right|$ that is distributed in CTA $q$, where CTA $q \in T_{i}$. Clearly, the summation of all $\left|r_{\phi(i)}\right|{ }^{(q)}$ 's is equal to $\left|r_{\phi(i)}\right|$. For the example of Figure $5(\mathrm{c})$, consider $\left(r_{\phi(1)}\right.$, $\left.r_{\phi(2)}, r_{\phi(3)}\right)=(\mathrm{NRT} 1, \mathrm{NRT} 2$, NRT 3$)$ and then $T_{1}=\{\mathrm{CTA} 3\}, T_{2}=\{\mathrm{CTA} 1, \mathrm{CTA}$

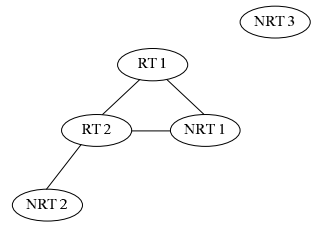

(a)

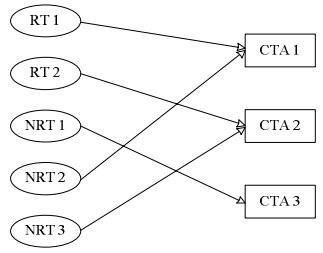

(b)

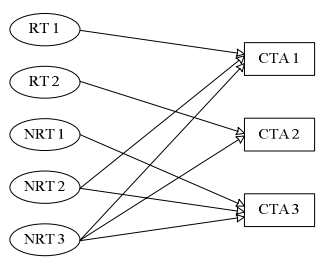

(c)

Fig. 5. An example.(a) The interference among requests.(b) The assignment after the grouping component.(c) The reassignment of NRT requests 
$3\}$ and $T_{3}=\{\mathrm{CTA} 1$, CTA 2, CTA 3$\}$. The distribution of $\mid$ NRT 1|, |NRT 2| and $\mid$ NRT $3 \mid$ is illustrated in Figure 6.

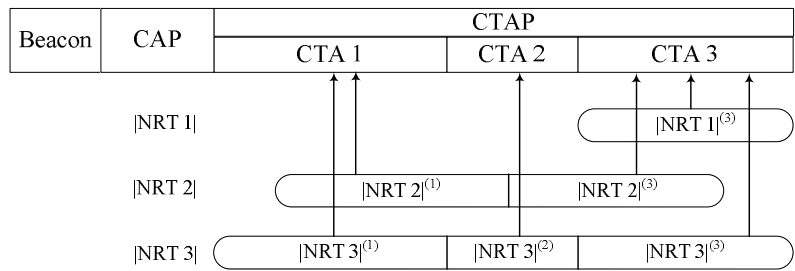

Fig. 6. The distribution of $\mid$ NRT 1|, |NRT 2| and |NRT 3|

Suppose that $k$ CTAs are used after the grouping component. We let $U$ denote the upper bound and $L_{j}$ denote the lower bound for CTA $j$, where $1 \leq j \leq k$. The goal of the BETM is to maximize $\sum_{i=1}^{m}\left|r_{\phi(i)}\right|$ subject to some constraints as follows. First, $\mid$ CTA $j \mid \geq L_{j}$ assures that the bandwidth requirements for the RT requests that are scheduled in CTA $j$ can be guaranteed. Second, $B+$ $|\mathrm{CAP}|+\sum_{j=1}^{k}|\mathrm{CTA} j| \leq U$ assures the maximal delay of any MAC frame is not greater than the maximally allowable length of the superframe, where $B$ is the length of the beacon and $|\mathrm{CAP}|$ is the length of the CAP. In other words, the delay limitations of all RT requests are promised.

Third, $\sum_{\mathrm{CTA}} j \in T_{i}|\mathrm{CTA} j| \geq\left|r_{\phi(i)}\right|$ and $|\mathrm{CTA} j| \geq\left|r_{\phi(i)}\right|^{(j)}$ assure that the bandwidth requirement of $r_{\phi(i)}$ can be satisfied. Finally, $\sum_{\mathrm{CTA}} \operatorname{liT}_{i}\left|r_{\phi(i)}\right|^{(j)}=$ $\left|r_{\phi(i)}\right|,\left|r_{\phi(i)}\right|^{(j)} \geq 0$ and $\left|r_{\phi(i)}\right| \geq 0$ are required. To sum up, the optimization problem can be expressed as a linear programming as follows.

$$
\text { Maximize } \sum_{i=1}^{m}\left|r_{\phi(i)}\right|
$$

subject to

$$
\begin{gathered}
|\mathrm{CTA} j| \geq L_{j} \text { for } 1 \leq j \leq k \\
B+|C A P|+\sum_{j=1}^{k}|\mathrm{CTA} j| \leq U \\
\sum_{\mathrm{CTA} j \in T_{i}}|\mathrm{CTA} j| \geq\left|r_{\phi(i)}\right| \text { for } 1 \leq i \leq m
\end{gathered}
$$

$|\mathrm{CTA} j| \geq\left|r_{\phi(i)}\right|^{(j)} \quad$ for $1 \leq i \leq m$ and every CTA $j \in T_{i}$

$$
\sum_{\mathrm{CTA} j \in T_{i}}\left|r_{\phi(i)}\right|^{(j)}=\left|r_{\phi(i)}\right| \quad \text { for } 1 \leq i \leq m
$$

$$
\begin{gathered}
\left|r_{\phi(i)}\right|^{(j)} \geq 0 \quad \text { for } 1 \leq i \leq m \text { and every CTA } j \in T_{i} \\
\left|r_{\phi(i)}\right| \geq 0 \text { for } 1 \leq i \leq m
\end{gathered}
$$


The optimization problem can be solved by several well-known methods, inclusive of the simplex method [12, the Karmarkar's interior point method 21, the ellipsoidal calculus method [18] and the Lagrange multiplier method [4]. They all can solve a linear programming efficiently.

\section{Simulation Results}

In this section, the performance of the proposed mechanism is evaluated by simulation. The Network Simulator (ns2) 3 are adopted for the simulation and the used IEEE 802.15.3 MAC functions are implemented by INTEL [13]. The simulation environment models a piconet which covers a $10 \mathrm{~m} \times 10 \mathrm{~m}$ area, where the DEVs are randomly distributed. Each DEV is equipped with a radio transceiver that is capable of transmitting up to 10 meters over a wireless channel. Each DEV can adjust its transmitting power dynamically. Since the design purpose of IEEE 802.15.3 is for the use of a WPAN, it is assumed that every transmitting pair has a distance of no more than 1 meter. The transmission capability of each network interface is assumed $100 \mathrm{Mbps}$.

There are three CBR flows (a type of RTs) in the simulation scenario whose bandwidths are $32 \mathrm{Kbps}, 1.5 \mathrm{Mbps}$ and $15 \mathrm{Mbps}$, respectively, and whose delay limitations are set to $45 \mathrm{~ms}, 75 \mathrm{~ms}$ and $90 \mathrm{~ms}$, respectively. In order to observe their QoS guarantee, up to ten FTP flows (a type of NRTs) are fed when the simulation proceeds. Since no scheduling method is specified in the IEEE 802.15.3 standard, first-come-first-served (FCFS) is implemented for comparison with the proposed mechanism. In the implementation, a traffic (RT or NRT) request will be rejected if it causes the total length of the beacon, the CAP and the CTAP exceeding $65535 \mathrm{~ms}$.

As observed from Figure 7, each CBR flow has a smaller packet delay than the delay limitation. The reason is that a packet whose delay exceeds the delay limitation is dropped. The influence of FTP flows on the packet delay for the proposed mechanism is negligible, which is a consequence of an upper bound

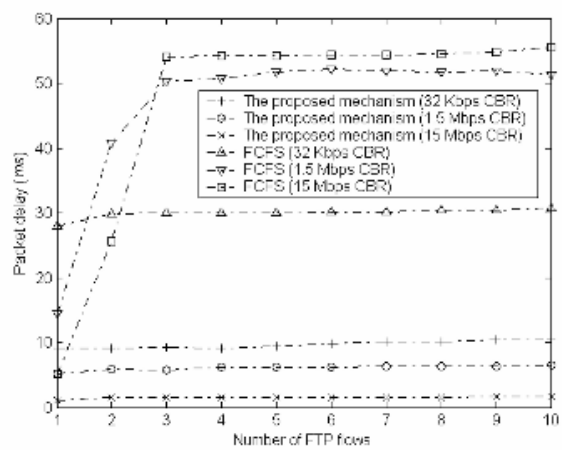

Fig. 7. Packet delay

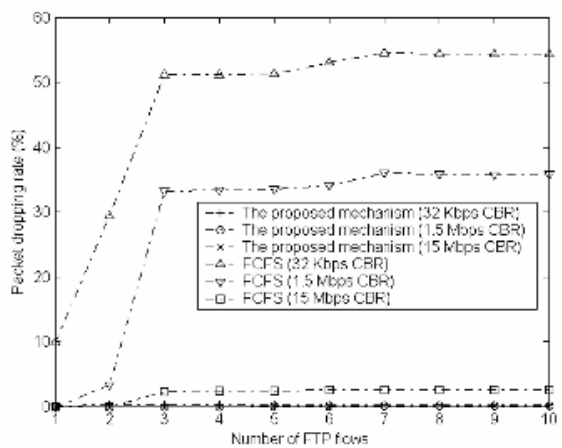

Fig. 8. Packet dropping rate 


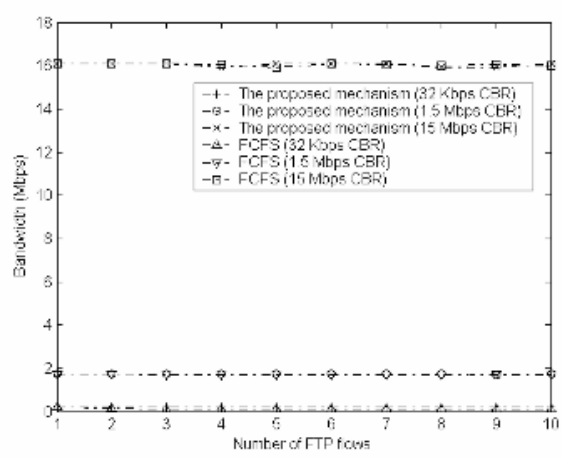

Fig. 9. Bandwidth

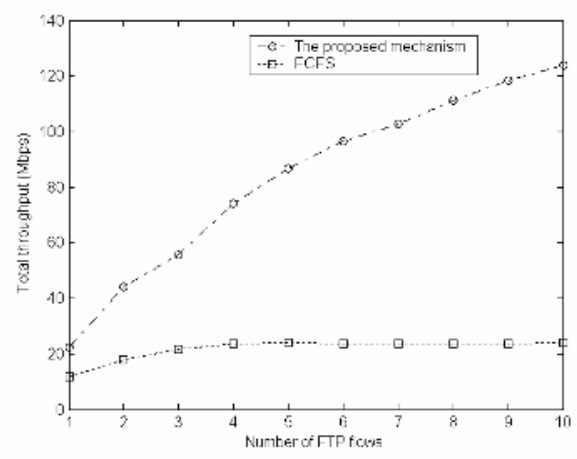

Fig. 10. Total throughput of NRTs

(obtained in Section 4) on the length of each superframe. The packet delay for FCFS is growing when the number of FTP flows is increasing until the bottleneck (three FTP flows in the simulation scenario) is reached.

Simulation results for packet dropping rate are shown in Figure 8. The packet dropping rate for the proposed mechanism approaches zero because the upper bound is not greater than the minimal delay limitation. All packets in the proposed mechanism are sent out before their delay limitations. Similar to Figure 7, the packet dropping rate for FCFS is growing when the number of FTP flows is increasing until the bottleneck is reached. Since the proposed mechanism results in a very low dropping rate, it can support multimedia traffics very well.

Simulation results for bandwidth are shown in Figure 9. Both the proposed mechanism and FCFS can guarantee the bandwidth requirement. Since IEEE 802.15.3 is TDMA based, it can guarantee the bandwidth requirement if there are enough CTAs for each RT. Both the proposed mechanism and FCFS can provide enough CTAs for each RT.

Simulation results for the total throughput of NRTs are shown in Figure 10, where each total throughput sums up the throughputs of all FTP flows. The total throughput for the proposed mechanism is much better than the total throughput for FCFS, which is a consequence of the grouping component and BETM component. After the grouping component, non-interfering FTP flows are allowed to be transmitted simultaneously, and after the BETM component, the total transmitting time of all FTP flows is maximized by applying any LP solution method. The total throughput for FCFS remains a constant when the number of FTP flows exceeds the bottleneck.

Finally, the numbers of accepted RTs for the proposed mechanism and FCFS are compared. Figure 11 shows the simulation results, where the bandwidth of the RTs is set to $15 \mathrm{Mbps}$. Since the proposed mechanism can transmit noninterfering RTs in parallel, it behaves better than FCFS. 


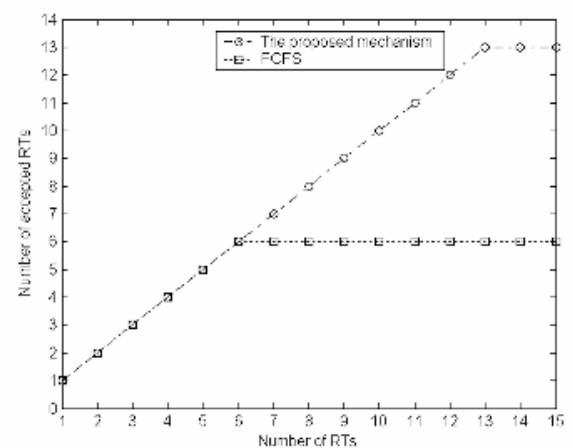

Fig. 11. Number of accepted RTs

\section{Conclusion}

In this paper, we have given an overview of the IEEE 802.15.3 MAC protocol and a description of UWB (IEEE 802.15.3a) technology. Both are greatly helpful to the future development of WPAN. An effective scheduling method is important for the IEEE 802.15.3 MAC to support novel applications in the future WPAN. Therefore, we proposed a resource allocation mechanism for the IEEE 802.15.3 MAC which contains a scheduling method. The effectiveness of the proposed mechanism was demonstrated by simulation. Since UWB technology can provide more accurate location information, it was used to improve the channel utilization.

The proposed mechanism comprises three components: grouping, CAC and BETM. The grouping component scheduled non-interfering traffics in the same CTA and attempted to minimize the number of CTAs used in the superframe. We proved that the problem of grouping is NP-hard, by showing that it is equivalent to the graph coloring problem. There are some existing algorithms, exact or approximate, for solving the graph coloring problem.

The CAC component performed admission control for QoS guarantee. The strategy is to compare the summation of all lower bounds for CTAs with the upper bound for the superframe. If the former exceeds the latter, it means that the available channel time is not adequate for RT requests and so the PNC has to reject an RT request. The BETM component scheduled NRTs so that their total throughput was maximized. To increase the total throughput, each NRT was scheduled to more CTAs as long as no interference occurred. The resulting optimization problem was formulated as an LP problem.

There are three advantages for the proposed mechanism. First, it can provide higher spatial reuse. By adjusting transmitting power, non-interfering traffics can be transmitted simultaneously. Second, it can guarantee the QoS properties of multimedia traffics. This was accomplished by the aid of lower bounds for all CTAs and the upper bound for the superframe, which were estimated according to the bandwidth requirements and delay limitations of RTs. Third, it 
can maximize the total throughput of NRTs. The maximization was based on a rescheduling of NRTs and an LP formulation.

\section{Acknowledgment}

This work was supported by the MediaTek Inc. under the project "Wireless Communication Systems". This work was also supported by National Science Council of Taiwan under the NSC93-2524-S-008-002 Integrated knowledge Management Project.

\section{References}

1. IEEE standard 802.15.3: Wireless Medium Access Control (MAC) and Physical Layer (PHY) Specifications for High Rate Wireless Personal Area Networks (WPANs), Inst. Elec. Electron. Eng., New York, USA, 2003.

2. FCC Notice of Proposed Rule Making, Revision of Part 15 of the Commission's Regarding Ultra-wideband Transmission Systems, ETDocket 98-153.

3. Ns2, the VINT project, http://www.isi.edu/nsnam/ns/.

4. G. Arfken, "Lagrange multipliers," in Mathematical Methods for Physicists, Academic Press, pp. 945-950, 1985.

5. A. BLUM, "New approximation algorithms for graph coloring," Journal of the ACM, vol. 41, pp. $470-516,1994$.

6. D. Brélaz, "New methods to color the vertices of a graph," Communications of the ACM, vol. 22, pp. 251-256, 1979.

7. P. Bhagwat, P. Bhattacharya, A. Krishma and S. Tripathi, "Enhancing throughput over wireless LANs using channel state dependent packet scheduling," Proceedings of IEEE INFOCOM, vol. 3, pp. 1133-1140, 1996.

8. J. C. R. Bennett and H. Zhang, "WF2Q: worst-case fair weighted fair queueing," Proceedings of IEEE INFOCOM, vol. 1, pp. 120-128, 1996.

9. H. M. Chaskar and U. Madhow, "Fair scheduling with tunable latency: a roundrobin approach," IEEE/ACM Transactions on Networking, vol. 11, pp. 592-601, 2003.

10. S. Capkun, M. Hamdi and J. P. Hubaux, "GPS-free positioning in mobile ad-hoc networks," Proceedings of the 34th Annual Hawaii International Conference, pp. 3481-3490, 2001.

11. A. Demers, S. Keshav, and S. Shenker, "Analysis and simulation of a fair queueing algorithm," Proceedings of ACM SIGCOMM, vol. 19, pp. 1-12, 1989.

12. G. B. Dantzig, "Programming of interdependent activities: II. mathematical model," Econometrica, vol. 17, pp. 200-211, 1949.

13. M. Demirhan, IEEE 802.15.3 MAC model for ns-2, http://www.winlab.rutgers. $\mathrm{edu} / \sim$ demirhan, Intel corporation.

14. J. Foerster, E. Green, S. Somayazulu and D. Leeper, "Ultra-wideband technology for short- or medium-range wireless communications," Journal of Intel Technology, 2nd quarter, 2001.

15. P. Goyal, H. Vin, and H. Cheng, "Start-time fair queueing: a scheduling algorithm for integrated services packet switching networks," IEEE/ACM Transactions on Networking, vol. 5, pp. 690-704, 1997. 
16. S. Golestani, "A self-clocked fair queueing scheme for broadband applications," Proceedings of IEEE INFOCOM, vol. 2, pp. 636-646, 1994.

17. M. M. Hallórsson, "A still better performance guarantee for approximate graph coloring," Information Processing Letters, vol. 45, pp. 19 -23, 1993.

18. A. B. Kurzhanskiand and I. Vályi, Ellipsoidal Calculus for Estimation and Control, MA: Birkhäuser: Boston, 1996.

19. D. Karger, R. Motwani and M. Sudan, "Approximate graph coloring by semidefinite programming," Journal of the ACM, vol. 45, pp. 246-265, 1998.

20. H. Karloff, Linear Programming, Birkhauser: Boston, 1991.

21. N. Karmarkar, "A new polynomial-time algorithm for linear programming," Combinatorica, vol. 4, pp. 373-395, 1984.

22. R. M. Karp, "Reducibility among combinatorial problems," Proceedings of a symposium on the Complexity of Computer Computations, pp. 85-104, 1972.

23. S. Lu, T. Nandagopal and V. Bharghavan, "Fair scheduling in wireless packet networks," Proceedings of ACM MOBICOM, vol. 27, pp. 63-74, 1997.

24. I. Méndez Díaz and P. Zabala, "A Branch-and-Cut Algorithm for Graph Coloring," proceedings of Computational Symposium on Graph Coloring and Generalizations, 2002.

25. T.S. Ng, I. Stoica and H. Zhang, "Packet fair queueing algorithms for wireless networks with location-dependent errors," Proceedings of IEEE INFOCOM, vol 3, pp. 1103-1111, 1998.

26. A. Parekh and R. Gallager, "A generalized processor sharing approach to flow control in integrated services networks: the single node case," IEEE/ACM Transactions on Networking, vol. 1, pp. 344-357, 1993.

27. H. A. Peelle, "Graph coloring in J: an introduction," Proceedings of ACM SIGAPL APL Quote Quad Conference, vol. 31, pp77-82, 2000.

28. P. Ramanathan and P. Agrawal, "Adapting packet fair queueing algorithms to wireless networks," Proceedings of ACM MOBICOM, pp. 1-9, 1998.

29. H. Zhang, "Service Disciplines for Guaranteed Performance Service in Packetswitching Networks," proceedings of the IEEE, vol. 83, pp. 1374-1396, 1995. 